\title{
«Décide de demeurer saisi de la question»
}

La mobilisation du Conseil de sécurité de l'ONU face aux crises

"Decides to Remain Seized of the Matter": The Mobilization of the United Nations Security Council Facing Crises

\section{David Ambrosetti}

\section{(2) OpenEdition}

\section{Journals}

\section{Édition électronique}

URL : http://journals.openedition.org/conflits/17728

DOI : $10.4000 /$ conflits. 17728

ISSN : $1777-5345$

Éditeur :

CCLS - Centre d'études sur les conflits lilberté et sécurité, L'Harmattan

Édition imprimée

Date de publication : 10 décembre 2009

Pagination : 99-122

ISBN : 978-2-296-11477-7

ISSN : $1157-996 X$

\section{Référence électronique}

David Ambrosetti, « «Décide de demeurer saisi de la question» », Cultures \& Conflits [En ligne], 75 | automne 2009, mis en ligne le 20 janvier 2011, consulté le 30 mars 2021. URL : http:// journals.openedition.org/conflits/17728; DOI : https://doi.org/10.4000/conflits. 17728 


\title{
« Décide de demeurer saisi de la question»
}

\author{
La mobilisation du Conseil de sécurité de l'ONU \\ face aux crises
}

\section{David AMBROSETTI}

Docteur en science politique (Centre d'étude d'Afrique noire, IEP de Bordeaux), David Ambrosetti est chargé de recherche au CNRS, à l'Institut des sciences sociales du politique (Université de Paris Ouest Nanterre La Défense). Il enseigne à l'Université Paris Ouest, à l'Université libre de Bruxelles (REPI), ainsi qu'à l'Université de Montréal (CERIUM), et collabore en outre avec les revues Cultures \& Conflits et Politique africaine. Ses travaux portent sur les normes sociales des diplomates et fonctionnaires en charge des conflits armés dans les institutions internationales (ONU et Union africaine). Il est l'auteur de Normes et rivalités diplomatiques à l'ONU : le Conseil de sécurité en audience, Bruxelles, P.I.E. Peter Lang, 2009. Contact : dambrosetti@u-paris10.fr

$\mathrm{O}$ bserver le fonctionnement des organisations internationales face aux situations qu'elles qualifient de «crises » est une façon d'étudier ce qui constitue une part importante, et croissante, des pratiques politiques déployées sur la scène internationale. Dans cet article, nous nous interrogeons sur les conditions - empiriquement sondées - qui permettent à une organisation intergouvernementale comme l'Organisation des Nations Unies (ONU) d'agir dans le domaine dit de la paix et la sécurité - selon la catégorisation indigène partagée par un large ensemble d'agents étatiques et de personnels d'organisations intergouvernementales et non gouvernementales, qui gravitent autour de ce domaine d'activité. Cela revient à comprendre comment l'ONU parvient à mobiliser des ressources matérielles et symboliques pour peser sur les choix des gouvernements visés par cette action internationale, et ce faisant, à pérenniser ses activités et ainsi contribuer à assurer sa survie comme institution. Mais nous tentons de comprendre ces conditions sans assimiler a priori les individus que nous avons observés à des organisations données ni à des propriétés prêtées à ces dernières, qu'il s'agisse de l'ONU elle-même (selon certaines interprétations fonctionnalistes ou institutionnalistes du multilatéralisme) ou de l'Etat que ces individus servent (interprétations réalistes). 
Pour ce faire, nous reconstituons dans le présent article des données empiriques recueillies lors d'une expérience d'observation participante au Conseil de sécurité de l'ONU, sur un mode ethnographique [cf. encadré ci-contre]. Ces données ont fait l'objet de croisements et de recoupements réalisés au fil de nos travaux ultérieurs sur les pratiques diplomatiques dans les opérations de paix de l'ONU, comme éléments d'une sociologie des professionnels internationaux de la paix.

Grâce à cette participation directe au travail quotidien d'une délégation siégeant au Conseil, nous avons pu reconstituer les règles, formelles et surtout informelles, reconnues par ces professionnels dans leur travail routinier de régulation des conflits armés au Conseil.

Plus précisément, ces règles ainsi reconstituées ont trait à la question particulière de la mobilisation du Conseil de sécurité sur un dossier spécifique. Elles participent bien de la grammaire de l'engagement des diplomates sur des dossiers de conflits armés. A travers leur examen, nous tentons de mieux comprendre les logiques de situation (systèmes de contraintes et d'opportunités qui s'imposent aux perceptions et pratiques des individus) ${ }^{1}$ dans lesquelles sont pris les agents diplomatiques siégeant au Conseil, et ce dans la relation que ces individus entretiennent non seulement avec leur organisation bureaucratique hiérarchisée (appareil diplomatique des Etats membres du Conseil de sécurité) mais aussi avec cette arène multilatérale que constitue le Conseil de sécurité, composante d'une autre organisation bureaucratique hiérarchisée, internationale cette fois : l'ONU.

La présente contribution ne prétend pas faire l'inventaire de la totalité des routines et règles formelles et pratiques reconnues par les agents eux-mêmes dans leur travail quotidien en matière de mobilisation du Conseil. Elle se limite à tirer certains enseignements quant aux conditions favorables ou défavorables à l'engagement du Conseil de sécurité sur des situations candidates à la qualification de « crises ».

En ce sens, ce travail espère éclairer le contenu que ces agents diplomatiques sont susceptibles de donner à cette notion de crise, notion qui échappe à toute taxinomie «technicienne » 2 . Ces crises qui rythment la vie des membres du Conseil de sécurité doivent en partie leur existence aux façons de faire et autres routines professionnelles propres à cet espace diplomatique multilatéral à l'ONU; les unes sont constitutives des autres, et réciproquement.

1. Dobry M., «Ce dont sont faites les logiques de situation », in Favre P., Fillieule O. et Jobard F. (dir.), L'atelier du politiste. Théories, actions, représentations, Paris, La Découverte, 2007, p. 119-148.

2. Buchet de Neuilly Y., «La crise ? Quelle crise ? Dynamiques européennes de gestion de crises ", in Le Pape M., Siméant J. et Vidal C. (dir.), Crises extrêmes : face aux massacres, aux guerres civiles et aux génocides, Paris, La Découverte, pp. 270-286. 


\section{Une observation participante au Conseil de sécurité de l'ONU}

Les données empiriques ici reconstitués ont été collectées à partir d'une période d'observation participante réalisée à la Mission permanente de la France à l'ONU, à New York, de juin à août 2002. Alors étudiant inscrit en doctorat, nous avons fait partie du " contingent » de stagiaires que la délégation française à New York accueille chaque année, dans différents services, pour des durées variant de trois-six mois à un an en moyenne. Ces contingents sont essentiellement fournis par les grandes écoles nationales (École nationale d'administration, Institut d'études politiques de Paris, etc.).

La délégation française faisait alors partie (pour ne pas préjuger de l'époque actuelle) des délégations recevant le plus de stagiaires à I'ONU, avec les délégations américaine et britannique, ainsi que la délégation de la Commission européenne. A ces stagiaires des délégations des Etats membres s'ajoutaient les nombreux stagiaires du Secrétariat de I'ONU. Au sein de la délégation française, nous étions le seul doctorant à cette date, et parmi les rares à étudier en " province » (Université Bordeaux IV / IEP de Bordeaux) : nous étions issu de la "filière bordelaise », pour reprendre le ton amusé du représentant permanent adjoint de l'époque, qui nous présentait à l'ambassadeur.

Il fut convenu que nous assurerions le suivi de dossiers de conflits armés africains, sous la responsabilité des deux diplomates en charge de ces questions. Cette position supposait la participation régulière aux débats sur ces dossiers à la table du Conseil de sécurité. Nous nous asseyions alors à la place dédiée à la délégation française, derrière la personne de la délégation chargée de parler au nom de la France (le représentant permanent, ou son adjoint, ou les responsables de notre stage lors de réunions de comités plus techniques, comme les comités de suivi de sanctions). Nous avions la charge de préparer, en lien avec nos responsables, les éléments de langage pour le représentant permanent et les télégrammes diplomatiques relatant les réunions du Conseil à destination des ministères concernés à Paris. Cela supposait le suivi de la documentation officielle (rapports du Secrétaire général, essentiellement) et " grise " concernant ces dossiers, ainsi des discussions informelles avec nos collègues étrangers, statutaires ou stagiaires suivant les mêmes dossiers que nous.

Dans ce travail, nous étions encadré par les deux responsables de notre stage, ce qui nous permit l'apprentissage de règles formelles et informelles propres au Conseil de sécurité et à la diplomatie française. Cet apprentissage s'exerçait dans un premier temps via les modifications apportées à nos rapports et notes, puis au travers d'échanges plus réguliers au cours desquels ces diplomates nous exposaient leurs analyses et nous invitaient à adopter et prolonger leurs raisonnements, à partager leurs préoccupations. L'organisation du travail interne à la délégation conduisait également à des échanges réguliers avec d'autres de ses membres, en marge des réunions quotidiennes autour de I'ambassadeur. Nous profitions de ces relations de travail pour rappeler à nos interlocuteurs, à l'occasion, notre position d'apprenti chercheur, en leur demandant des entretiens approfondis sur des points directement liés à nos recherches. Dans ces entretiens, nous restions à leurs yeux - nous a-t-il semblé - des membres de la délégation, certes novices, mais " de la maison », unis par une communauté d'objectifs et d'expériences. En dépit des satisfactions réelles que nous procurait le travail réalisé, notamment certaines discussions de stratégie politique " dans le feu de I'action », nous conservions à l'esprit nos ambitions de chercheur, et restions davantage préoccupé par la maturation de l'analyse politologique que le doctorant que nous étions devait être en mesure d'offrir sur le " marché académique » à partir de cette expérience (ce qui nous prédisposait par exemple à consacrer du temps et de l'énergie à questionner en silence les choses apparemment les plus banales), que par notre aptitude à endosser pleinement et entièrement notre rôle de stagiaire. Notons enfin que, conformément au règlement du stage, nous ne restituons dans ce texte aucune information directement tirée des dossiers spécifiques dont nous avions la charge durant notre stage. 
Décomposer les règles pratiques, les normes partagées par ces agents diplomatiques dans leur travail quotidien, revient donc à comprendre les ressorts par lesquels un conflit est transformé en dossier de crise requérant un traitement plus ou moins urgent (la qualification de crise ne suffisant pas en soi à inspirer l'urgence, et l'urgence ne garantissant pas toujours le traitement le plus « efficace » dudit dossier).

Pour entrer dans le vif de notre sujet, reprenons un élément de la liturgie propre au Conseil de sécurité de l'ONU, le dernier alinéa de toutes les résolutions du Conseil : " Décide de demeurer saisi de la question ». Cette phrase entend traduire l'engagement du Conseil sur un dossier donné, mais ne permet pourtant pas de préjuger de l'intensité et de la durée de cet engagement. Certaines conditions doivent être préalablement satisfaites pour que le Conseil puisse se déclarer ainsi saisi d'une question.

Avant l'examen de ces conditions empiriques de l'engagement du Conseil de sécurité, nous discutons les choix méthodologiques qui guident ce travail en faveur d'une sociologie des pratiques diplomatiques en partie nourrie par une expérience ethnographique originelle (I). L'examen de ces conditions de mobilisation du Conseil débute ensuite par une première présentation des règles formelles et informelles autour desquelles s'organise le travail de mobilisation du Conseil (c'est-à-dire la constitution d'un dossier de conflit armé dont les membres du Conseil sont saisis). Ces règles et normes sont ici regroupées selon les phases successives du travail du Conseil sur un dossier donné (II). Mais ces conditions de mobilisation de l'ONU via son Conseil de sécurité incluent également la « grande » question des «intérêts » portés par les diplomates sur ces dossiers de conflits précis. L'article propose ici une grille d'analyse et d'interprétation de ces intérêts. Celle-ci conduit à rechercher des groupes d'interaction et de socialisation plus restreints au sein du travail quotidien des représentants permanents au Conseil, et à déterminer les positions reconnues aux agents diplomatiques dans ces groupes, les attentes collectives afférentes à ces positions, et les risques que ces attentes font peser sur ces diplomates, sur les ressources sociales qu'ils captent dans ces groupes (III).

\section{Pour une sociologie de la pratique diplomatique à l'ONU}

Nombreuses sont les entreprises théoriques dans la discipline des relations internationales qui visent à définir les unités d'analyse les plus pertinentes (Etats, «civilisations », « individus » sur le modèle de l'bomo œeconomicus, firmes et autres acteurs transnationaux, ou - plus rarement - classes sociales) et à modéliser le système international à partir de la généralisation de modèles de comportement individuels relativement simples, directement inspirés de la théorie sociale ou de la théorie économique. Leur objectif reste toujours le même : «expliquer » pourquoi la politique internationale est ce qu'elle est ${ }^{3}$. Elles laissent en ce sens peu de place à l'investigation empirique des pro- 
cessus qui se jouent en deçà. Pour le dire brutalement, les individus spécifiques qui peuplent les « cas d'étude » choisis comme «tests empiriques » à des raisonnements quasi exclusivement hypothético-déductifs intéressent finalement assez peu en tant que tels, de même que les pratiques précises qu'ils portent, les ressources grâce auxquelles ils agissent, et les groupes précis auxquels ils sont liés et qui leur fournissent ces ressources.

On le sait, cet excès de positivisme a généré une puissante réponse critique prompte à débusquer le pouvoir qui imprègne - et se dissimule dans - les structures sociales sous-tendant les interactions entre ces unités, c'est-à-dire en deçà de ce que les acteurs poursuivent et rationalisent en tant qu'unités du système, et en deçà même du système que constituent ensemble ces unités ${ }^{4}$. La structure sociale la plus visée par ces approches fut sans nul doute le langage, diffuseur privilégié de catégories mentales réifiant des rapports de force au profit des vainqueurs du moment.

On peut toutefois s'inquiéter, avec Iver Neumann, de la profusion de travaux portant sur les discours des décideurs politiques et diplomatiques - ou de tout autre acteur de la scène politique internationale - sans égard pour les diverses autres pratiques qui accompagnent ces discours (ces derniers constituant eux-mêmes des ensembles de pratiques) ${ }^{5}$. Après un "linguistic turn » accompagnant le «constructivist turn » des années 1990, un «practice turn " 6 est apparu comme une nécessité à des auteurs de la discipline inspirés par la sociologie ou l'anthropologie, et souvent issus de ces disciplines. La restitution des pratiques de hauts fonctionnaires nationaux et internationaux incluant les pratiques verbales - est précisément l'ambition première de cet article.

Cette ambition suppose des choix méthodologiques. Essentiellement inductive, la méthode ethnographique apparaît au premier abord comme la plus à même de révéler les pratiques sociales à l'état brut, permettant à l'anthropologue de rattacher ensuite ces pratiques aux récits et mythes fondamentaux qui les supportent au sein du groupe social considéré, de la culture considérée. Incidemment, elle offre à l'observateur l'espace de liberté nécessaire pour la restitution des conditions dans lesquelles il a opéré ses observations.

3. Rappelons le débat des années 1990 entre Alexander Wendt et Kenneth Waltz, tous deux appliquant aux relations politiques et de sécurité entre Etats des modèles de comportement généraux déduits de la théorie sociologique interactionniste de l'École de Chicago pour le premier et de la théorie économique néoclassique libérale pour le second.

4 . Pour une illustration récente de ces approches structurales et des reproches qu'elles adressent aux approches positivistes, voir Joseph J., « Hegemony and The Structure-Agency Problem in International Relations: A Scientific Realist Contribution ", Review of International Studies, vol.34, n¹, 2008, pp. 109-128.

5. Neumann I. B., "Returning Practice to the Linguistic Turn: The Case of Diplomacy ", Millennium, vol.31, $\mathrm{n}^{\circ} 3,2002$, pp. 627-651.

6. Schatzki T. R., Knorr-Cetina K. et von Savigny E., The Practice Turn in Contemporary Theory, New York, Routledge, 2001. 
Iver Neumann offre une belle illustration d'un regard ethnographique porté sur les diplomates comme groupe relativement cohérent, doté d'une "culture » spécifique ${ }^{7}$. Cette approche convient d'autant mieux aux éléments empiriques reconstitués dans notre présent article qu'il s'agit ici d'observations personnelles réalisées lors d'une période d'immersion dans cet environnement « culturel » particulier. Ces observations ont permis d'identifier, par inférences, certaines règles du jeu diplomatique au Conseil de sécurité de l'ONU, des règles que nous avons ultérieurement testées et validées grâce à l'approfondissement d'opérations spécifiques de l'ONU, en recourant à des entretiens avec des élites diplomatiques, à l'étude des travaux spécialisés et à l'analyse du traitement médiatique.

Toutefois, la présente étude ne correspond que très imparfaitement à une ethnographie des diplomates siégeant au Conseil de sécurité. Wanda Vrasti appelle à une certaine prudence à l'encontre des vertus que des auteurs des Relations internationales reconnaissent aujourd'hui à l'ethnographie - inspirant un nouveau «tournant » dans la discipline, ethnographique celui-là 8 . En effet, selon Vrasti, on tend à associer au regard ethnographique trois caractéristiques pourtant contestées au sein même de l'anthropologie contemporaine : 1/ la pureté d'une collecte d'informations supposée vierge de tout prisme interprétatif préalable, source de données authentiques et précises ; 2/ un mode de restitution de ces informations permettant à l'observateur d'énoncer son expérience d'observation telle qu'il l'a vécue, facilitant ainsi l'effort de réflexivité de sa part ; 3/ une préférence pour une théorisation qui se fonde sur les pratiques les plus quotidiennes et non sur les grandes interprétations discursives du politique que les Relations internationales affectionnent tant 9 . Les quelques illustrations proposées par l'auteur ${ }^{10}$ soulignent en effet combien ces trois caractéristiques sont fortement postulées, alors même que l'ethnographie demeure une écriture, une production textuelle de connaissance, chargée de représentations particulières de l'expérience qui ne peuvent être réduites à une simple question de méthode ${ }^{11}$.

Les cadres interprétatifs préexistants doivent donc faire l'objet d'un effort réflexif propre, que la méthode ethnographique ne garantit pas en soi. L'analyse des diplomates du Conseil de sécurité que nous présentons ici répond ainsi à un questionnement spécifique, qui trouve sa source dans la tradition sociologique. Ce questionnement concerne les modalités de reconnais-

7. Neumann I. B., « Returning Practice», art.cit, ou encore « "A Speech That the Entire Ministry May Stand for”, or: Why Diplomats Never Produce Anything New », International Political Sociology, vol.1, n², 2007, pp. 183-200.

8. Vrasti W., "The Strange Case of Ethnography and International Relations ", Millennium, vol.37, n`2, 2008, pp. 279-301.

9. Ibid., p. 281.

10. Ces illustrations sont puisées chez Carol Cohn, Cynthia Enloe, Vincent Pouliot et Iver Neumann.

11. Vrasti W., art.cit., pp. 293-294. 
sance de l'influence entre pairs dans l'espace diplomatique international, en milieu multilatéral en particulier, et ce dans le domaine de la régulation collective de la violence armée. Quelles normes sociales régulent la reconnaissance de l'influence, l'acceptation collective d'une position d'influence et des pratiques de pouvoir qui s'y rattachent, lorsqu'il s'agit de traiter des violences armées d'autrui, c'est-à-dire des pratiques de conquête du pouvoir et de gouvernement considérées comme les plus violentes 12 ?

Pour satisfaire cette ambition théorique, la sociologie nous semble fournir les meilleurs outils, à travers le lien qu'elle permet d'établir entre les pratiques individuelles, les ressources sociales fournies par les groupes dont les individus font partie, et les normes dominantes reconnues par ces groupes en matière d'usage des ressources qu'ils fournissent. Et nous suivons pleinement Peter Jackson lorsqu'il note, à propos de la sociologie de Pierre Bourdieu et l'usage de la notion de culture en histoire internationale, la capacité de la théorie sociologique de surmonter, dans la pratique même de la recherche, la dichotomie entre unité et système, entre agent et structure, une vertu très appréciable dans une discipline des Relations internationales aux postulats souvent si (inutilement) tranchés dans ce débat ${ }^{13}$. Dans le présent travail, la sociologie fournit les outils intellectuels permettant de contextualiser, de socialiser des interactions observées : quelles règles, quelles ressources, avec quels effets politiques?

\section{La mobilisation du Conseil de sécurité : de l'inscription à l'ordre du jour à l'adoption d'un texte}

Grâce à la participation directe au travail quotidien d'une délégation siégeant au Conseil de sécurité, nous avons pu reconstituer quelques règles formelles et informelles reconnues par les professionnels de la diplomatie multilatérale dans leur travail routinier au Conseil. Classiquement, nous choisissons de restituer ces règles en les regroupant selon les phases successives qui ponctuent le travail du Conseil sur une situation de « crise ».

Nous n'oublions pas ce que cette présentation séquentielle a de discutable : elle assimile chaque situation de «crise » nouvelle, chaque « dossier » nouveau, à une pièce vide dans laquelle se réuniraient subitement tous les agents intéressés par ladite situation, et seulement eux, pour n'échanger que sur les enjeux liés à cette situation, dans les limites géographiques et temporelles strictes de cette «crise », en excluant du décor les trajectoires de ces

12. Qu'on nous permette de renvoyer ici à deux récents travaux explicitant ce questionnement et les recherches empiriques qui en découlent: Ambrosetti D., Normes et rivalités diplomatiques à l'ONU. Le Conseil de sécurité en audience, Bruxelles, P.I.E. Peter Lang, 2009 ; et "Urgences et normalités de gestionnaires face aux violences "des autres" : l'ONU et le Soudan ", Actes de la recherche en sciences sociales, n¹74, septembre 2008, pp. 81-99.

13 . Jackson P., "Pierre Bourdieu, the 'cultural turn' and the practice of international history ", Review of International Studies, vol.34, n¹, 2008, pp. 155-181. 
agents et leurs relations préalables potentielles, y compris sur d'autres situations voire sur de tout autres sujets et enjeux. Nous n'oublions pas non plus ce que les trajectoires personnelles de ces agents diplomatiques peuvent enseigner sur ces normes de la diplomatie multilatérale onusienne, sur leur reproduction et leurs transformations. La description séquentielle nous semble néanmoins offrir le mode de restitution le plus lisible pour ces normes que nous avons reconstituées à partir des observations de terrain réalisées, certes au prix d'une mise entre parenthèses de l'effort réflexif de l'observateur.

\section{L'inscription à l'ordre du jour}

C’est à la délégation qui préside le Conseil pour un mois qu'il incombe d'établir l'ordre du jour durant le mois de sa présidence. Une grande partie de cet ordre du jour s'impose de fait au président, puisqu'elle relève de questions déjà ouvertes et qui sont soumises à réexamen régulier selon un calendrier préétabli, en particulier pour le renouvellement des mandats des opérations de paix de l'ONU au moment où ils arrivent à leur terme. Toutefois, le président du Conseil peut inscrire de nouveaux sujets, voire retarder certains points initialement prévus au profit de sujets qu'il juge brûlants.

Pour l'inscription d'une nouvelle question, néanmoins, le président doit obtenir l'accord de chacun des autres membres du Conseil. C'est là une règle coutumière, qui nous a été rapidement indiquée par les diplomates qui nous encadraient, en vertu de laquelle une seule délégation peut formellement bloquer le Conseil de sécurité. Bien entendu, le refus initial d'une ou plusieurs délégations ne signifie pas l'abandon du projet, mais bien le commencement de tractations entre délégations pour convaincre le ou les réfractaires.

Souvent, ces tractations prennent la forme d'un échange de bons procédés. En effet, une délégation souhaitant cette inscription à l'ordre du jour pourra s'adresser directement aux réfractaires pour leur demander de changer de posture, se plaçant ainsi en position de débiteur vis-à-vis des seconds, qui ne manqueront pas de le rappeler lorsqu'un sujet les intéressera fortement. La forte institutionnalisation des relations entre les délégations des Etats membres permanents - due à une présence constante au Conseil - explique la fréquence de ce type de transactions.

Cependant, d'autres ressorts classiques de la diplomatie peuvent intervenir : la mise en jeu de relations de bonne entente, des mesures d'incitations ou de rétorsions, y compris sur d'autres dossiers, ce qui rappelle l'importance de mesurer le degré de fongibilité des ressources utilisées dans les différents domaines de politique internationale ${ }^{14}$, dans la diplomatie multilatérale en particulier.

14. Cox R., «Social Forces, States, and World Orders: Beyond International Relations Theory », 
Une solution de compromis relativement répandue consiste à entamer une première discussion sur le sujet controversé à la table du Conseil lorsque sont abordés les " points divers ", en fin de séance. Ensuite, au cas où une délégation souhaiterait soumettre un texte au vote des membres du Conseil - une déclaration présidentielle qui sera lue par le président du Conseil à l'issue de la séance, ou une résolution du Conseil - le sujet pourra être traité sous des points génériques déjà prévus à l'ordre du jour (par exemple le point «paix et sécurité en Afrique ", régulièrement traité au Conseil). Durant le printemps 2008, les délégations américaine et britannique du Conseil ont ainsi obtenu la discussion d'une résolution visant à condamner le Zimbabwe pour les violences d'Etat qui ont accompagné les élections de mars 2008, au point « paix et sécurité en Afrique », vu les réticences d'autres membres (en particulier l'Afrique du Sud) d'inscrire la question à l'ordre du jour. D'ailleurs le projet de résolution n'a pas été formellement soumis au vote, étant donné l'opposition de ces mêmes Etats relayés par la Chine et la Russie ${ }^{15}$.

Cela renvoie à l'étape suivant l'inscription d'une question à l'ordre du jour : la discussion des textes par lesquels le Conseil prend position sur cette question et, le cas échéant, met en place des dispositifs d'action.

\section{La discussion des textes}

Le Conseil décide et agit au travers de textes élaborés puis votés par ses membres. Avant leur adoption, ces textes font l'objet de discussions, selon des modalités relativement routinières et formalisées.

En général, une délégation s'engage davantage que les autres dans la rédaction du texte, et dans les tractations informelles, parce qu'elle s'intéresse davantage au sujet, et/ou parce qu'on lui reconnaît une prééminence sur ce dossier : les diplomates disent alors que cette délégation « a le lead » sur ce dossier ${ }^{16}$. Selon cette division sociale informelle du travail parmi les diplomates travaillant au Conseil de sécurité, une délégation qui s'affaire pour obtenir de ses pairs l'inscription d'un dossier à l'ordre du jour peut ainsi être considérée comme une candidate au leadership sur ce dossier ${ }^{17}$. Qu'une telle position d'influence soit tacitement reconnue ou non, la ou les délégations qui ont sollicité la mobilisation de leurs partenaires sur un sujet ont le plus souvent l'initiative de rédiger les textes à discuter.

in Cox R. (avec Sinclair T.J.), Approaches to World Order, Cambridge University Press, 1996, p. 103.

15. Entretien avec un diplomate français en charge des questions onusiennes et multilatérales au ministère des Affaires étrangères, Paris, 30 juillet 2008.

16. Voir Ambrosetti D. et Cathelin M., "Les enjeux du leadership au Conseil de sécurité : responsabiliser ou contrôler les opérations de paix de l'ONU ? », Revue internationale et stratégique, $\mathrm{n}^{\circ} 68,2008$, pp. 69-79.

17 . Notons que sur les dossiers africains subsahariens, les candidats ne se bousculent généralement pas. 
La discussion des textes procède par consultations informelles. Le décor mérite ici d'être planté. La grande salle du Conseil de sécurité constitue le lieu où les décisions sont formellement prises. Elle est équipée d'une tribune pour accueillir des observateurs extérieurs (journalistes accrédités etc.) et de caméras filmant les interventions orales des délégations et leurs votes à main levée. Mais avant de procéder à un vote formel, les membres du Conseil s'engagent dans des échanges informels, très variables dans leur longueur et leur fréquence, selon les sujets. Ces consultations ont lieu dans une salle attenante à la grande salle. Cette salle des consultations accueille les quinze délégations (chacune dispose de trois chaises), mais aussi des personnes invitées à intervenir devant ces délégations (des membres du Secrétariat ou d'agences de l'ONU par exemple). Des chaises sont également disposées dans la salle pour accueillir là aussi un public, qui est bien plus restreint que dans la grande salle : il se compose d'autres membres des délégations siégeant au Conseil, mais aussi de certains membres du Secrétariat de l'ONU disposant des badges requis pour pénétrer dans cette salle, dont l'entrée est gardée par des policiers.

Parce que les agents diplomatiques des autres Etats de l'ONU et les membres d'ONG accréditées savent que les décisions se jouent dans cette salle, il est très fréquent de voir ces derniers attendre en nombre (en fonction de l'importance accordée au sujet discuté) dans le salon attenant à cette salle des consultations, afin de prendre connaissance de ce qui s'y est dit. Pour obtenir ces informations, ces agents exploitent les connaissances interindividuelles qu'ils entretiennent parmi les autres personnes autorisées à assister à ces consultations ${ }^{18}$.

La négociation se poursuit bien sûr à l'extérieur des lieux qui lui sont explicitement dédiés, en particulier dans les couloirs. Une autre pratique fréquente consiste à réunir, dans les missions permanentes des Etats membres, des délégations (siégeant ou non au Conseil de sécurité) qui partagent un intérêt commun à se concerter parallèlement à ces discussions au Conseil. Par exemple, la délégation de l'Etat qui préside pour six mois l'Union européenne organise fréquemment des réunions entre représentants permanents des autres Etats membres de l'Union juste avant les séances de consultations au Conseil. Ces réunions visent à rechercher sur ce sujet une position commune qui devra être soutenue par les Etats membres siégeant au Conseil. Les deux membres de l'Union qui disposent d'un siège permanent au Conseil ont certes montré qu'ils restaient, en matière de politique étrangère, attachés à une certaine indépendance vis-à-vis du reste de l'Union, et qu'ils devaient prendre en compte d'autres considérations liées à leur statut de membre permanent.

18. Gare à ceux qui succomberaient à la somnolence durant ces séances de consultations : à peine sortis de la salle des consultations, ils pourraient se trouver assaillis par une foule pressée de rapporter à sa hiérarchie le contenu des échanges tenus lors de ces consultations, comme l'a expérimenté l'auteur de ces lignes. Pour poursuivre l'anecdote, voir Alain Dejammet, Supplément au voyage en Onusie, Paris, Fayard, 2003, sur les aspects cocasses du quotidien à l'ONU, et notamment sur les lieux prédisposant à la somnolence. 
Lors de ces tractations, encore une fois, tous les leviers diplomatiques «traditionnels » restent disponibles. Au plan «bilatéral » (voire interindividuel) en marge de ces consultations, on pensera à l'échange de bons procédés, à la mise en jeu de relations de bonne entente, sans oublier la menace de mesures de rétorsions (par exemple la mise en jeu d'une future adhésion à une alliance militaire ou à une organisation multilatérale autre que l'ONU ${ }^{19}$ ). Mais le « jeu » se joue également au cœur de la discussion multilatérale, à travers l'effet que les négociateurs peuvent produire sur leurs pairs et sur le cours de la négociation. Cela pousse les diplomates à consacrer une énergie considérable à l'élaboration d'arguments juridiquement inattaquables et/ou politiquement acceptables.

A l'appui de leurs arguments, ces diplomates invitent d'ailleurs régulièrement des représentants d'Etats non membres du Conseil mais directement concernés par la question débattue pour qu'ils s'expriment devant le Conseil, sans droit de vote ${ }^{20}$, ou encore des représentants d'organisations spécialisées sur la question et/ou agissant sur le terrain. A ce propos, invoquer l'existence de mouvements de plaidoyer et de mobilisation publique relayés par les médias internationaux constitue parfois une véritable ressource pour agir sur les négociateurs ${ }^{21}$. Ce qui laisse d'ailleurs entrevoir l'importance des relations entretenues par une délégation diplomatique à New York avec des journalistes et avec des organisations engagées dans le plaidoyer. L'effet des médias sur le travail diplomatique doit donc faire l'objet d'une analyse fine, loin des interprétations superficielles du fameux «CNN effect » 22 .

\section{Le vote}

Ces négociations visent à obtenir un texte dont on espère le vote unanime ${ }^{23}$. Mais cet objectif souffre d'un nombre croissant de blocages, qui caractérise les relations aujourd'hui difficiles entre les diplomaties les plus interventionnistes au sein du Conseil de sécurité en matière de régulation des conflits armés (Etats occidentaux) et celles qui y sont hostiles au nom du prin-

19. L’information circule rapidement entre délégations dans ces cas. Ce fut le cas après que le représentant permanent des États-Unis sous l'administration de Georges W. Bush, John Negroponte, eut averti le représentant bulgare, Stefan Tafrov, que la Maison Blanche s'opposerait probablement à l'adhésion de la Bulgarie à l'OTAN si cette dernière ne soutenait pas la position américaine favorable à une immunité permanente des personnels des opérations sous mandats de l'ONU à l'égard de la Cour pénale internationale. Voir Ambrosetti D., «S'opposer aux États-Unis au Conseil de sécurité. L'argumentation contre la puissance dans les négociations multilatérales ", Études internationales, vol.35, n`3, 2004, p. 475.

20. En application de l'article 37 du règlement intérieur provisoire du Conseil.

21. Ambrosetti D., «S’opposer aux États-Unis au Conseil de sécurité », art.cit.

22. Robinson P., The CNN effect: the myth of news, foreign policy and intervention, London, New York, Routledge, 2002.

23 . Dans leurs télégrammes, notes écrites et interventions orales, les diplomates français rappellent régulièrement leur attachement à cette quête d'unanimité au Conseil, au nom de la préservation de l'autorité de cette institution qui occupe une place cruciale dans le dispositif d'influence internationale de la diplomatie française. Cet élément de la «culture » diplomatique française mériterait certes une analyse systématique quant à sa réalité et ses traductions 
cipe de la souveraineté des Etats ${ }^{24}$. Dans les faits, l'objectif reste d'obtenir le vote positif de neuf membres, sans aucun vote négatif provenant d'un membre permanent. La position de chaque membre est connue lorsque les délégations quittent la salle des consultations pour se rendre dans la grande salle du Conseil afin de passer au vote formel en séance publique. Le président du Conseil attend en effet de recevoir l'aval des délégations pour passer à cette phase, qui consiste en un affichage public des positions de chacun. Une ou plusieurs délégations peuvent souhaiter ne plus discuter le texte et passer rapidement au vote formel en dépit d'une configuration défavorable au texte apparue dans les intentions de vote. La manoeuvre vise ici à intimider les récalcitrants déclarés et les inciter à voter finalement en faveur du texte, ou à les contraindre à afficher publiquement leur opposition. Mais dans la très grande majorité des cas, la séance publique sert à l'adoption formelle d'un texte, c'est-à-dire à un vote favorable, quand bien même ce texte aurait perdu une bonne part de sa substance par rapport à ce que souhaitaient ses défenseurs. Dans ces conditions, sauf exceptions ${ }^{25}$, il est inutile de passer en séance publique pour obtenir le rejet du texte. Cette séance n'aura pas lieu et l'on saura grâce aux bruits de couloir ce qui a empêché l’adoption du texte.

\section{Traduire les décisions $d u$ Conseil en actes}

Lorsqu'elles ne sont pas strictement déclaratoires, les décisions du Conseil de sécurité s'imposent au Secrétariat de l'ONU chargé de les exécuter, ce qui explique d'ailleurs la parole qui est régulièrement donnée aux fonctionnaires du Secrétariat, aussi bien à la table du Conseil en séances de consultation et en séances publiques, que dans les couloirs et les missions permanentes au moment de la rédaction des textes. Le Secrétariat fait le lien avec les dimensions opérationnelles et avec les différents partenaires (contributeurs de troupes, bailleurs de fonds, services juridiques, gestion des ressources humaines, agences opérationnelles de l'ONU, ONG « sous-traitantes », etc.) ${ }^{26}$.

contemporaines. De façon significative, un diplomate étatsunien a pu soutenir devant nous l'importance qu'il accordait au contraire à l'existence de positions tranchées et de votes serrés au Conseil comme signe de «bonne santé démocratique » de cette institution multilatérale. Entretien avec un diplomate en charge des questions africaines à l'ambassade des États-Unis à Paris, 15 décembre 2005.

24. Ce constat, fréquent dans les milieux diplomatiques, semble avoir également marqué l'envoyée spéciale et le correspondant du quotidien Le Monde à la dernière Assemblée générale de l'ONU ; voir Nougayrède N. et Bolopion P., «A l'ONU, les Occidentaux aux prises avec les puissances émergentes ", Le Monde, 22 septembre 2008.

25. Rappelons les vifs débats qui ont précédé l'offensive armée des États-Unis contre l'Irak en 2003, qui ont donné l'occasion à des élites diplomatiques et politiques de premier plan d'expliquer les raisons de leur rejet du texte soumis par la délégation américaine au vote des membres du Conseil de sécurité.

26. Howard L., UN Peacekeeping in Civil Wars, Cambridge, Cambridge University Press, 2008, p. 340 ; Jenkins R., "The UN Peacebuilding Commission and the Dissemination of International Norms ", Crisis States Research Centre Working paper n³8, Londres, Development Studies Institute, London School of Economics, juin 2008, p. 6. 
A ce stade, les membres du Conseil n'interviennent en tant que tels que dans le suivi des actions menées par le Secrétariat, et ce, essentiellement de deux façons. La première est l'examen, à dates préétablies, des rapports d'étape du Secrétaire général qui détaillent les types d'intervention et les évolutions de la situation. Ces rapports sont ainsi fréquemment attendus avant l'échéance du mandat d'une opération, afin de déterminer l'opportunité ou non de le renouveler. La seconde suppose une implication accrue des membres dans des groupes informels constitués pour le suivi de dossiers donnés (groupes d'amis du Secrétaire général 27, comités de suivi des régimes de sanction décidés par le Conseil à l'encontre de responsables politico-militaires, etc.).

\section{La fin de l'engagement}

Il reste enfin à évoquer la dernière phase du processus de mobilisation du Conseil : la fin de l'engagement onusien. Cette phase est significative : il n'existe aucune formule faisant le pendant du « décide de demeurer saisi de la question » pour sanctionner officiellement la sortie de jeu du Conseil de sécurité. Autrement dit, les délégations membres n'annoncent jamais - à notre connaissance 28 - qu'elles décident de dessaisir le Conseil d'une question, de clore un dossier ouvert à sa table. Le sujet disparait simplement de l'ordre du jour. Mais formellement, il n'est jamais clos par les membres du Conseil.

Bien sûr, les décisions de redéployer ou de mettre un terme des opérations de paix de l'ONU ne sont pas rares, quels que soient les résultats de ces opérations. Il en fut ainsi le 30 juillet 2008, lorsque le Conseil mit fin à la l'UNMEE, la mission de paix déployée en 2000 à la frontière entre l'Érythrée et l'Éthiopie. Ces décisions sont facilitées par la forme des mandats octroyés aux missions de paix de l'ONU. Après l'ONUC au Congo de 1960 à 1964, des délégations mécontentes de la gestion très volontariste de cette opération par le Secrétaire général Dag Hammarskjöld plaidèrent avec succès pour l'adoption de mandats à durée limitée (6 mois) renouvelables ${ }^{29}$. Ainsi, en cas d'absence de décision (par impossibilité d'obtenir une majorité qualifiée ou du fait d'un veto d'un membre permanent), le mandat de l'opération expire automatiquement. A ce stade, déployer à nouveau des Casques bleus sur ce même terrain exigera l'adoption d'une nouvelle résolution fixant le mandat de ce qui sera une nouvelle opération. A l'approche de l'échéance du mandat, la possibilité de «mettre en péril » une opération de paix constitue un levier de pression sur le Conseil, y compris sur de tout autres sujets que la situation liée à

27. Whitfield T., Friends Indeed? The United Nations, Groups of Friends, and the Resolution of Conflict, Washington, United States Institute of Peace, 2007.

28 . Cette observation tirée de nos expériences et de nos recherches nous a été confirmée par un diplomate français en charge des questions onusiennes et multilatérales au ministère des Affaires étrangères. Entretien, Paris, 30 juillet 2008.

29. Bellamy A., Williams P. et Griffin S., Understanding Peacekeeping, Cambridge, Polity, 2004, pp. 71-73. 
cette opération précise ${ }^{30}$. Notons ici l'existence d'une possibilité de " prorogation technique » (row over) du mandat dont le renouvellement serait en cours de négociation. Cette solution permet aux membres du Conseil d'éviter qu'une force de l'ONU se retrouve déployée sur le terrain illégalement (car sans mandat) en cas de désaccord persistant pour le renouvellement de son mandat.

En conclusion, l'apparition d'une « crise » à la table du Conseil ne saurait résulter de la seule « irruption » de violences à grande échelle dans telle ou telle partie du globe. Elle naît de l'intervention - plus ou moins formellement codifiée - d'agents diplomatiques siégeant au Conseil de sécurité et de leur travail de mise en forme institutionnelle. Avec comme conséquence la possibilité que des conflits armés restent « orphelins » ${ }^{31}$, en cas de désintérêt de chacun des membres. La constitution d'un dossier conduisant à la mobilisation du Conseil doit donc être interprétée dans la relation entre ce que les délégations « veulent » traiter et ce qu'elles «ne pourraient pas » ne pas traiter, selon les canaux divers qui assurent la médiation de ces «volontés » et de ces " nécessités » politiques.

L'examen de ces quelques règles formelles et informelles autour desquelles s'organise le travail diplomatique au Conseil de sécurité ne suffit pas, néanmoins, à comprendre les formes et les degrés de mobilisation du Conseil de sécurité face aux situations urgentes faisant partie de ses domaines d'attribution. Mais il permet de mieux éclairer l'aspect plus «substantiel » de la mobilisation : l'intérêt à agir des représentants permanents des Etats membres, c'est-à-dire les liens qu'ils établissent entre les situations dont ils sont saisis, les objectifs qu'ils sont censés atteindre, et les possibilités que leur offre leur présence au Conseil de sécurité pour atteindre ces objectifs.

\section{Les diplomates à l'ONU et leurs "intérêts » : quelles sanctions sociales en jeu face aux crises?}

L' « intérêt » à mobiliser ou non l'ONU demande à être mieux compris. Assimiler l'intérêt des ambassadeurs, engagés dans une série de choix contextualisés au sein Conseil de sécurité, à celui de l'Etat - l' « intérêt national » des présentations réalistes canoniques de la politique internationale - est une facilité, potentiellement trompeuse ${ }^{32}$, que nous tentons d'écarter a priori. L'observation de praticiens dans leurs relations avec leurs homologues à

30. Comme l'a illustré la délégation américaine en mettant en péril la MINUBH (Mission de l'ONU en Bosnie-Herzégovine) en juin et juillet 2002 afin d'obtenir le vote d'une immunité des personnels sous mandat de l'ONU vis-à-vis de la Cour pénale internationale. Ambrosetti D., «S’opposer aux États-Unis », art.cit.

31. Pour reprendre une formule tirée de la littérature « experte » sur les conflits. Voir Whitfield T., Friends Indeed ?, op. cit., p. 7.

32. Guzzini S., "The Enduring Dilemmas of Realism in International Relations ", European Journal of International Relations, vol.10, ${ }^{\circ} 4,2004$, pp. 533-568. 
l'ONU, mais également avec leurs pairs et leur hiérarchie, nous a rapidement encouragé à étendre nos questionnements sociologiques à cette " grande » question des relations internationales, celle des intérêts des acteurs observés à la table du Conseil. Quelques pistes empiriques concernant la mobilisation du Conseil de sécurité face aux conflits armés voudront en montrer l'utilité potentielle.

Une interprétation de la décision par les normes? La pratique diplomatique et sa sanction sociale

Lorsqu'elles délimitent et formalisent ce qu'est « leur » intérêt dans une négociation multilatérale sur une question donnée, les élites diplomatiques ne nous semblent pas faire autre chose que rechercher dans leur environnement les indications leur permettant d'ajuster leurs pratiques à ce qui serait jugé le plus normal, le plus adéquat.

On retrouve là un axiome fondamental des approches constructivistes en relations internationales. Selon cet axiome, directement inspiré par la sociologie (interactionniste et structurationniste en particulier), les agents qui interagissent sur la scène internationale ou contribuent à l'actualisation de pratiques internationales recherchent avant tout, comme les autres agents sociaux, à produire l'action appropriée, l'action reconnue comme normale, c'est-à-dire collectivement attendue et sanctionnée dans l'environnement social de ces agents. Autrement dit, les identités de ces agents (appartenance à tel ou tel groupe) et les positions qu'ils occupent au sein de ces groupes déterminent les normes que ces agents intériorisent au cours de leur socialisation et à partir desquelles ces agents définissent les pratiques les plus appropriées, pour y ajuster en permanence leurs propres pratiques ${ }^{33}$.

Comme nous l'avons développé plus longuement ailleurs, cet axiome, pour constituer un outil potentiellement heuristique dans la recherche empirique, mérite selon nous d'être sérieusement complexifié, contre une interprétation par trop rigide et bien souvent idéaliste, qui s'est diffusée dans la littérature spécialisée. Nous nous limitons à exposer ici, sans autre démonstration, quatre amendements que nous lui avons apportés ${ }^{34}$.

33. Les références classiques liées à cette vulgate constructiviste renvoient par exemple aux travaux de March J. et Olsen J., ou encore à l'article de synthèse de Jepperson R. L., Wendt A. et Katzenstein P. J., « Norms, Identity and Culture in National Security » in Katzenstein P. (dir.), The Culture of National Security, New York, Columbia University Press, 1996.

34 . Pour une critique de cette interprétation figée et idéaliste du schéma sociologique constructiviste, et pour l'élaboration de notre démarche dans un va-et-vient permanent entre théorie et recherche empirique, voir Ambrosetti D., Normes et rivalités diplomatiques à l'ONU, op. cit., notamment le chapitre I, « Normes sans éthique. Les enjeux pratiques de la sanction sociale », pp. 31-77. 
D’abord, cet environnement social ne saurait être considéré comme un tout unifié et harmonieux. Il doit être décomposé en groupes précis, ce qui renvoie aux appartenances multiples des agents sociaux et aux positions diverses qu'ils occupent d'un groupe à l'autre.

Deuxièmement, les membres de chacun de ces groupes sont unis par des liens plus ou moins institutionnalisés et formalisés. Autrement dit, ils peuvent constituer un groupe, producteur d'une appartenance, sans nécessairement se le dire avec des mots, sans nommer ce groupe et cette appartenance. Et il en est de même des positions que ces agents se reconnaissent les uns à l'égard des autres au sein dudit groupe. De telles positions n'ont pas besoin d'un haut degré de formalisation, voire d'une rationalisation verbale, pour exister comme positions. Le raisonnement s'applique enfin aux normes attachées à ces appartenances et à ces positions, qui là encore peuvent exister hors de toute formalisation et même de toute rationalisation verbale.

Troisième point, si l'adoption des pratiques jugées appropriées dans un groupe donné résulte d'une intériorisation de ses normes, une telle intériorisation ne nécessite pas l'adhésion auxdites normes (fondée en logique et/ou en éthique) ${ }^{35}$. La force de la répétition, en particulier de la répétition des sanctions sociales positives et négatives adressées par les membres du groupe à chacun d'entre eux, est un moteur puissant de socialisation, donc d'intériorisation des normes du groupe. L'analyse sociologique doit donc conduire à l'identification des pratiques de sanction sociale positive et négative produites de façon répétée par ledit groupe à l'égard de ses membres selon des paramètres spécifiques.

Quatrième et dernier point, l'importance de se conformer aux normes d'un groupe (en fonction de la position que chaque agent occupe dans ce groupe) risque trop souvent d'être postulée sans vérification empirique approfondie. Or, une façon de l'établir est de mesurer l'importance que l'agent accorde au groupe en question (parmi les multiples groupes dans lesquels il est amené à s'insérer du fait de sa position institutionnelle), en vertu de l'importance des ressources que ledit agent capte dans ce groupe, selon que ces ressources contribuent pour beaucoup ou non à la reproduction des positions institutionnelles (notamment décisionnelles) qu'il occupe, ou à la conquête de positions institutionnelles qu'il convoite. Il faut donc, pensons-nous, se garder d'établir un lien trop mécanique entre, d'une part, les identités et les positions des agents, et d'autre part, la conformité aux normes supposément attachées à ces identités et positions dans les groupes sociaux qui reconnaissent ces dernières. C'est là l'objet d'une investigation sociologique.

35. Et c'est heureux : démontrer qu'un agent social adhère à une idée est une tâche empiriquement glissante et qui devrait être abandonnée, comme l'ont récemment soutenu Krebs R.R. and Jackson P.T., "Twisting Tongues and Twisting Arms: The Power of Political Rhetoric ", European Journal of International Relations, vol.13, n¹, 2007, pp. 35-66. 
Cette grille d'analyse amendant le schéma constructiviste canonique conduit, au plan méthodologique, à questionner de la façon suivante les décisions d'un diplomate siégeant au Conseil de sécurité en matière de mobilisation du Conseil face à des situations de conflits armés : quelles sont les normes, c'est-à-dire les attentes collectives, qui sont susceptibles d'influer sur la façon dont le représentant permanent d'un Etat définit l'action appropriée face à la situation de conflit armé dont il est saisi en tant que membre du Conseil ? Pour le savoir, il faut identifier les groupes, parmi ceux qui reconnaissent à ce haut fonctionnaire ses identités et ses positions institutionnelles (positions qui lui permettent de siéger et d'agir au nom de la diplomatie de son Etat), qui sont susceptibles de remplir les deux conditions suivantes :

1) ils doivent constituer des groupes importants pour ce représentant permanent, dans l'économie de ses identités et positions ; l'importance de ces groupes pour notre diplomate renvoie à l'importance que ce dernier accorde aux ressources sociales et matérielles que ces groupes lui fournissent aujourd'hui, et/ou qu'ils lui ont fournies hier, et/ou qu'ils sont susceptibles de lui fournir demain ;

2) ces groupes doivent entretenir, à l'égard dudit représentant permanent siégeant au Conseil, des attentes collectives qui pourraient se trouver affectées par la décision de mobiliser ou non le Conseil sur des conflits armés en général, ou sur telle ou telle situation de conflit armé en particulier ; pour le dire autrement, la réponse apportée par notre diplomate à cet enjeu de mobilisation du Conseil doit avoir des répercussions prévisibles sur sa capacité à répondre aux attentes collectives dudit groupe.

Si ces conditions sont remplies, il nous semble fondé de poser comme hypothèse que les normes dudit groupe ont pu intervenir dans la façon dont ce représentant permanent a défini l'action appropriée et opté pour telles pratiques plutôt que telles autres. Les régularités observées au sein dudit groupe, dans les interactions entre ce haut fonctionnaire et les autres membres dudit groupe, doivent alors faire l'objet d'une attention particulière, en tant qu'enjeux de sanction sociale, et particulièrement en tant que source de risques de sanction négative, et ce en vertu des positions spécifiques reconnues à ce diplomate et à ses interlocuteurs au sein de ce groupe spécifique.

\section{Groupes sociaux et positions engagés dans le travail diplomatique à l'ONU}

Ainsi, la décision de mobiliser le Conseil de sécurité de l'ONU face à un conflit armé engage directement un premier groupe, qui réunit les représentants permanents des quinze Etats membres du Conseil au moment où la question est soulevée en son sein, et avec eux leurs délégations respectives en poste dans les missions permanentes de ces Etats membres à New York. C'est 
à ce groupe qu'il incombe de discuter et d'adopter, selon les règles décrites plus haut, les textes qui vaudront décision du Conseil, avec plus ou moins de conséquences institutionnelles pour les Nations Unies dans leur ensemble, et plus ou moins d'effets politiques sur les acteurs régionaux dudit conflit armé.

Cette position institutionnelle, chaque membre de ce groupe des représentants permanents la doit d'abord à sa hiérarchie, aux autorités politiques de son Etat. Parce que ces mêmes autorités politiques conservent le pouvoir de lui retirer cette position, il n'est pas a priori déraisonnable de postuler chez lui une grande sensibilité aux attentes portées par les membres de son gouvernement sur les sujets de conflits armés dont il a la charge à l'ONU. La chose n'en est que plus évidente lorsque ce représentant permanent doit sa nomination essentiellement aux relations qu'il a précédemment nouées avec des élites et des partis politiques, ou encore avec des courants intellectuels fortement liés à ces derniers. Son appartenance à ces groupes et les positions d'influence qu'il a su conquérir en leur sein détermineront les normes qui façonneront au quotidien ses pratiques diplomatiques, surtout sur les dossiers érigés en symboles par ces élites, partis ou courants (mettant par exemple en jeu une hostilité profonde ou une amitié sans faille à l'égard de tel ou tel régime ${ }^{36}$ ).

Les attentes de sa «capitale » ne se résument toutefois pas à celles de son seul gouvernement. Le processus d'ajustement des positions à défendre sur les sujets traités à l'ONU est complexe entre, d'une part, le responsable politique à la tête de la diplomatie (le ministre, lui-même sous l'autorité du chef de l'exécutif en matière de politique étrangère) et ses conseillers, et d'autre part, les professionnels de la diplomatie, qui demeurent au service de cette dernière indépendamment des alternances politiques à la tête de l'exécutif, en vertu d'une organisation administrative fortement bureaucratisée.

L'accession à cette position de représentant permanent à l'ONU, une position très prestigieuse et convoitée dans la diplomatie française, ne saurait être découplée de la reconnaissance dont les titulaires successifs de cette position ont bénéficié de la part de leurs pairs au sein de cette organisation bureaucratique. Cette reconnaissance se traduit, en France, par l'entrée dans le corps des ambassadeurs (un groupe réputé pour sa relative autonomie), puis par une ascension dans l'organigramme du ministère et dans la hiérarchie officieuse des directions, services et ambassades les plus convoités. Ce parcours professionnel au cœur de la diplomatie (en tant que corps professionnel et organisation bureaucratique) garantit, par divers dispositifs de sélection, la possession préalable de certaines qualités, de certains capitaux sociaux distinctifs. Il entretient en outre la diffusion de normes spécifiques, dont certaines interviennent directement dans la définition des intérêts, des « choses à faire " ${ }^{37}$. Une telle

36. Pensons, par exemple, aux divisions qu'on observe aujourd'hui parmi les élites diplomatiques françaises sur la question des positions à l'égard de l'adhésion de la Turquie à l'Union européenne, ou encore sur celle du nucléaire iranien. 
socialisation commune n'efface certes pas les marqueurs symboliques et les tensions normatives autour desquels différents groupes sociaux disposant de capitaux en partie différenciés se distinguent et s'opposent au sein même de leur corps professionnel ${ }^{38}$. Elle informe toutefois l'agent des attentes que ses pairs entretiennent à l'égard des positions qu'il peut convoiter, modelant ce faisant l'espace des possibles dans l'exercice de ces positions, une fois « conquises».

Décrire empiriquement la part de cette socialisation professionnelle et politique proprement nationale dans la détermination des pratiques diplomatiques dominantes à l'ONU dépasse le cadre des objectifs que nous nous sommes fixés dans cet article. Contentons-nous de relever que, en matière de régulation internationale des conflits armés, de nombreux choix opérés par le représentant permanent d'un Etat à l'ONU ne se jouent pas dans la relation qu'il entretient avec sa «capitale ». Elles relèvent souvent de son initiative et de son analyse de la situation et des intérêts à défendre dans ladite situation, et ses choix gagnent d'autant plus aisément l'assentiment de sa hiérarchie que cette dernière n'a guère d'expectative sur ce dossier précis. C'est précisément fréquemment le cas en matière de conflits armés en Afrique subsaharienne.

Dans cette initiative et cette analyse " propres » du représentant permanent, néanmoins, doivent être recherchés d'autres groupes et d'autres positions, qui se jouent cette fois "à l'international ", c'est-à-dire sur la scène diplomatique internationale elle-même, ou encore sur la scène transnationale. Au regard des ressources sociales non négligeables qu'elles peuvent procurer à un diplomate en poste à l'ONU, les relations nouées et durablement entretenues (vecteurs d'appartenance et de positionnement dans ces groupes) avec des réseaux de plaidoyer, think tanks internationaux - tels que l'International Crisis Group, par exemple -, firmes multinationales, associations professionnelles, groupes d'intérêts, etc., méritent d'être prises en compte dans l'identification des pratiques appropriées sur des situations précises.

Mais restons dans l'espace diplomatique onusien. L'Organisation des Nations Unies, avec ses logiques institutionnelles propres au multilatéralisme et aux bureaucraties internationales, produit des groupes d'appartenance et des positions d'influence différenciés, souvent peu formalisés. Le lead

37. A propos de la politique étrangère de la France vis-à-vis des États africains francophones nés des décolonisations sous la Ve République, on peut rappeler ici l'homogénéité des élites administratives qui, au sein de la présidence française et des ministères des Affaires étrangères et de la coopération, ont façonné cette politique. Meimon J., «Se découvrir militant. Le cabinet Cot à l'épreuve de la Coopération (1981-1983) », Politix, vol.18, n70, 2005, pp. 113-134. Ce phénomène semble connaître des transformations significatives depuis le début des années 1990.

38. Le bel exemple qui nous vient à l'esprit ne concerne toutefois pas la diplomatie mais le corps des officiers de l'armée de terre : Christel Coton, «Luttes de prestige entre pairs. Le cas des officiers de l'armée de terre », Sociétés contemporaines, n72, 2008, pp. 15-35. 
reconnu à un membre du Conseil sur un dossier donné ( $c f$. supra) constitue bien une de ces positions. Pensons également à l'appartenance aux groupes d'amis et autres groupes de contact en lien avec les activités du Secrétariat de l'ONU en matière de résolution des conflits, depuis l'élan donné à cette formule par Álvaro de Soto et Javier Pérez de Cuellar à la fin des années 198039. Ces groupes illustrent d'ailleurs tout l'intérêt et la difficulté d'analyser de telles positions informelles reconnues entre pairs, en ce qu'ils se constituent sui generis, par l'initiative d'Etats membres qui délimitent ensemble le périmètre du groupe qu'ils entendent former, d'où leur grande plasticité qui complique le travail de classification ${ }^{40}$.

Ce sont parfois même des carrières nouvelles qui s'ouvrent au contact du milieu onusien, ce qui laisse présager une influence plus grande des attentes portées dans ces arènes et dans les groupes qui les composent et les traversent. Ainsi, en particulier dans des Etats offrant peu de perspectives et d'avantages comparatifs à leurs diplomates, les ambassadeurs nommés au poste de représentant permanent à l'ONU à New York savent qu'ils peuvent espérer se distinguer auprès des diplomaties et des services les plus influents au sein de l'Organisation et se voir directement recrutés par l'Organisation à des conditions bien plus favorables. Pensons, par exemple (parmi bien d'autres), à l'ancien ambassadeur nigérian, Ibrahim Gambari, devenu Représentant spécial du Secrétaire général Ban Ki-moon.

Mais une telle promesse requiert de fournir un ensemble de garanties, de répondre à un ensemble d'attentes portées par ces acteurs les plus influents et/ou nombreux avec le consentement (ou la résignation) des autres. En cas d'échec, c'est non seulement la porte donnant accès à ces opportunités de carrière à l'ONU qui se ferme, mais c'est parfois le poste même d'ambassadeur à l'ONU qui est perdu. Il n'est en effet pas si rare que les dirigeants politiques ou les élites diplomatiques d'un Etat, souvent influent, exigent de leurs homologues d'un autre Etat, en général moins influent, qu'ils renvoient l'ambassadeur que ces derniers avaient choisi pour les représenter à l'ONU. Ainsi, agir dans le cadre de ce qui est attendu comme acceptable par ces décideurs influents fait partie des déterminants pour la suite de la carrière de cet ambassadeur dans son propre système diplomatique national ${ }^{41}$.

39. Whitfield T., Friends Indeed?, op. cit., p. 33.

40 . Ibid., p. 2.

41. Par exemple, Celso Amorim, une figure de la vie politique brésilienne (ministre des Affaires extérieures de 1993 à 1995) devenu représentant permanent du Brésil à l'ONU (1995-1999), aurait fait l'objet de telles demandes de la part des autorités politiques de son grand voisin du nord, agacées par les positions défendues par le premier à la table du Conseil à propos des sanctions contre l'Irak lorsque le Brésil siégeait au Conseil de sécurité (1998-1999) et que Celso Amorim présidait un panel au sein du Conseil traitant des conséquences humanitaires de ces sanctions. Entretien téléphonique avec un diplomate français en poste à l'ONU en 1999, juillet 2002. La candidature d'Amorim comme chef de la nouvelle Commission de contrôle, de vérification et d'inspection des Nations Unies pour le désarmement de l'Irak (COCOVINU), créée en décembre 1999, fut ainsi écartée par Kofi Annan, du fait de l'oppo- 
L'analyse des «intérêts » des diplomates siégeant au Conseil face aux situations de conflits armés dont ils sont saisis (par l'un d'entre eux, le plus souvent, comme nous l'avons vu plus haut) ne saurait procéder par seule déduction, tant ces intérêts peuvent s'avérer nombreux, divers, et parfois inattendus pour l'observateur. Cette analyse revient, dans notre optique, à établir une cartographie des attentes que des groupes spécifiques font peser sur eux au fil de leurs interactions - passées et présentes. Il s'agit plus précisément d'une cartographie des risques perçus par ces diplomates pour leurs ressources sociales et, à terme, pour les positions décisionnelles qu'ils occupent et/ou convoitent.

La mobilisation du Conseil au cour des conflits armés : une cartographie des attentes et des risques

Le schéma suivant [voir page suivante] reprend certaines de ces positions, identifiées dans nos recherches, et leurs attentes afférentes en matière de mobilisation du Conseil de sécurité face à une situation de conflit armé.

Quatre ensembles de positions méritent d'être approfondies. Une première position reconnue aux représentants permanents reprend un des éléments les plus récurrents en politique internationale, la fourniture de garanties de sécurité, ce qui signifie la reconnaissance collective des positions de protecteurs et de protégés entre diplomates en charge de leurs relations réciproques. Et il faut noter que ces positions valent également en ce qu'elles sont reconnues par les autres diplomaties, qui constituent de nouveaux protégés potentiels ou des protecteurs concurrents potentiels, sur la scène régionale à tout le moins. Cette relation protectrice, parfois à l'origine de zones d'influence quasi exclusive, facilitera l'engagement en faveur d'une mobilisation du Conseil s'il s'agit d'un régime protégé. Ou alors, la protection est assurée de façon bilatérale et le "patron » protecteur refuse au contraire toute immixtion extérieure. Et à l'inverse, si le «client » protégé n'est pas le régime menacé par la «crise » mais au contraire le « fauteur » de la «crise » (ou l'un des fauteurs), la position de protecteur pousse à refuser ou freiner une gestion internationale trop intrusive.

Mais d'autres positions reconnues aux élites diplomatiques tiennent plus directement au fonctionnement spécifique de la diplomatie multilatérale à l'ONU. Une deuxième position sociale que nous souhaitons pointer est celle réunissant les diplomaties qui se sont saisis des nouveaux récits de mobilisation diplomatique et juridique à l'ONU en matière de régulation internatio-

sition des délégations américaine et britannique, comme le rappellent l'article de Lynch C., «Ekeus Won't Be New Iraq Arms Monitor », Washington Post, 15 janvier 2000, p. A26, ainsi qu'une liste de discussion de l'organisation Campaign Against Sanctions on Iraq (disponible sur http://www.casi.org.uk/discuss/2000/msg00038.html, consultée le 5 novembre 2009). Cela n'empêcha pas Amorim de poursuivre sa carrière politique, et d'être nommé ministre des Affaires extérieures par le président Lula da Silva en 2003, puis confirmé en 2006. 


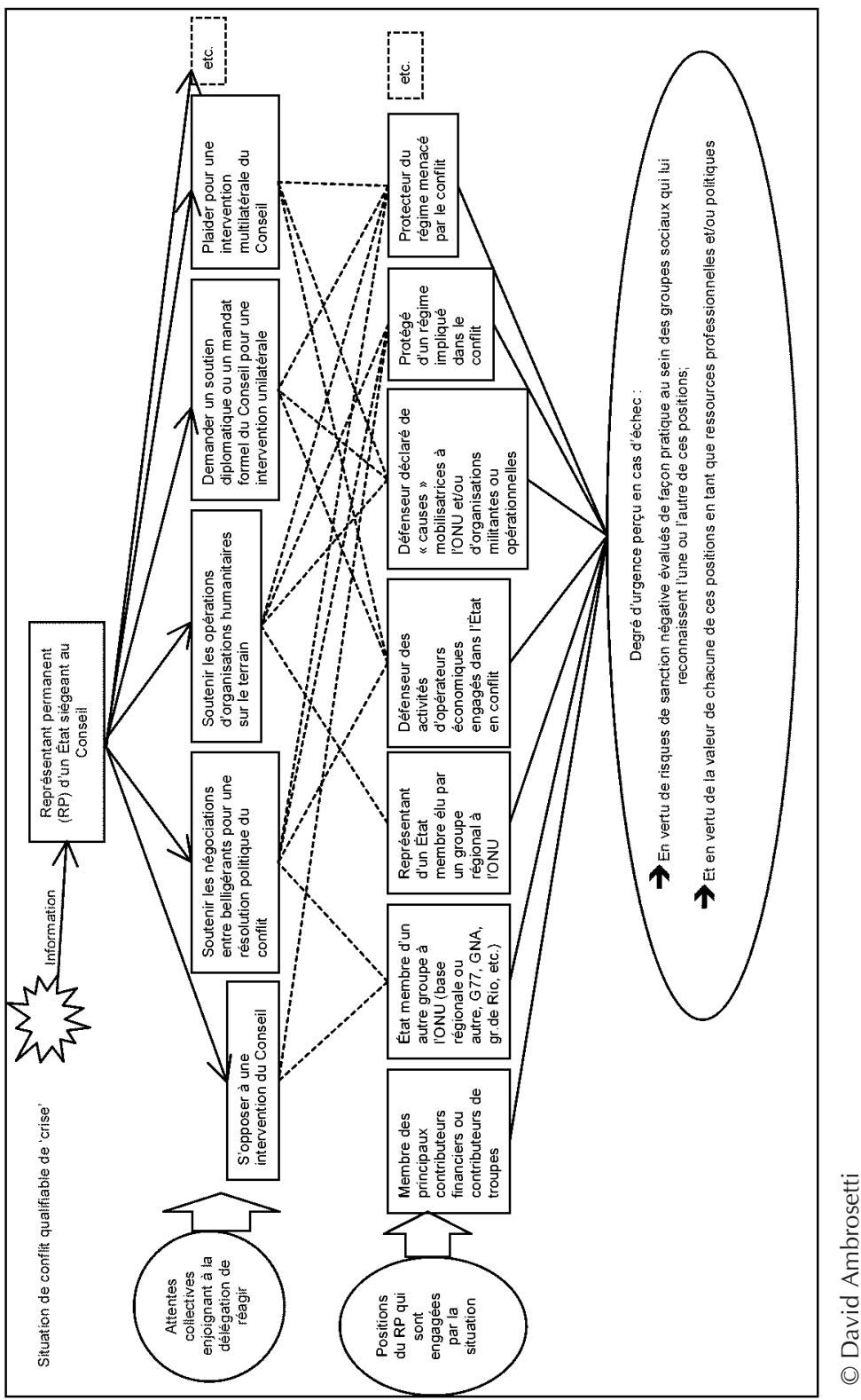


nale de la violence fondés sur un fort retentissement médiatique, dans les sociétés occidentales à tout le moins (discours humanitaire, sécurité humaine, responsabilité de protéger, etc.), ces diplomaties pouvant difficilement se désintéresser ouvertement des débats concernant ces récits. Une position connexe est, bien entendu, celle qui unit des décideurs diplomatiques à des organisations militantes ou opérationnelles dont ils sont issus, et qui restent soucieux de l'avenir des activités de ces organisations. Pensons au rôle d'un Robert Fowler, ambassadeur du Canada à l'ONU bruyamment engagé sur le nouveau front de la sécurité bumaine au cours des années 1990.

Un troisième ensemble de positions naît en particulier des relations de solidarité au sein de groupements d'Etats constitués sur une base régionale ou d'intérêts jugés communs et qui ont acquis une certaine épaisseur institutionnelle (autour du Groupe des 77 ou du Groupe des non alignés, par exemple, groupes dont on connaît les orientations de principe hostiles aux formes d'action les plus intrusives et coercitives en matière de résolution des conflits armés). En ce qui concerne les regroupements régionaux, nous avons déjà mentionné les efforts de coordination et de solidarité minimale entre délégations membres de l'Union européenne. On peut également évoquer le Groupe de Rio, ou les pays de l'ASEAN, ou de l'Union africaine, ou autre. Rappelons aussi les appartenances à des groupes d'amis et autres groupes de contacts sur des situations ou en faveur d'opérations données.

L'appartenance à des groupes régionaux concerne particulièrement les membres non permanents du Conseil de sécurité. Ces derniers sont en effet élus dans leur groupe régional au sein de l'Assemblée générale (1. Etats d'Afrique, 2. Etats d'Asie, 3. Etats d'Europe orientale, 4. Etats d'Amérique latine et des Caraïbes et 5. Etats d'Europe occidentale et autres Etats), après avoir été sélectionnés par le Conseil de sécurité parmi les candidats de leur groupe, et avant un vote formel à l'Assemblée. Ces candidats organisent donc de véritables campagnes électorales, orientées vers les membres du Conseil de sécurité (en particulier les permanents), mais aussi vers les membres de leur groupe régional. Cela suppose des garanties fournies à ces derniers en matière de régulation des crises et conflits armés ${ }^{42}$.

Enfin, le dernier ensemble de positions que nous voulons souligner reprend la division du travail propre au fonctionnement des opérations de paix de l'ONU. Deux ensembles d'Etats membres de l'ONU sont directement concernés par les projets d'opérations discutés au Conseil : ceux qui fournissent l'essentiel des troupes (personnels en uniforme) à l'ONU, et ceux

42. Par exemple, l'Union africaine a pris position en 2008 contre les procédures judiciaires pénales nationales ou internationales lancées par des États non africains contre des dirigeants politiques et des agents étatiques civils ou militaires africains, ce qui limite les choix des membres africains élus au Conseil de sécurité lorsque les projets de résolution évoquent le recours à de telles procédures judiciaires extra-africaines. 
qui fournissent les principaux efforts financiers pour la réalisation de ces opérations. A la suite des réformes concernant la conduite de ces interventions par le département des opérations de maintien de la paix au Secrétariat de l'ONU (rapport de Lakhdar Brahimi de novembre 2000), une place plus importante tend à être accordée aux principaux contributeurs de troupes (provenant essentiellement du sous-continent indien) en amont de la décision. On invite ces diplomaties à se prononcer à la table du Conseil de sécurité (sans droit de vote) lorsqu'elles n'y siègent pas. Plus récemment, la naissance de la commission de consolidation de la paix en juin 2006 a consacré ce principe, en réservant cinq sièges, parmi les trente et un qui composent la commission, aux Etats figurant parmi les dix premiers contributeurs de troupes de l'ONU 43. Une telle inflexion doit être rattachée aux nouvelles ressources politiques et matérielles à disposition de certains grands contributeurs devenus des puissances «émergentes », et à la nouvelle place qu'ils savent ainsi se ménager visà-vis de leurs pairs à l'ONU.

Cette même commission réserve également cinq sièges aux principaux donateurs volontaires pour l'appui financier des opérations de l'ONU. Ceuxci doivent être distingués des contributeurs financiers qui s'en tiennent à respecter leur quote-part dans le budget des opérations de paix. Ces quotes-parts se basent sur celles du budget ordinaire de l'ONU (avec une majoration pour les membres permanents), et sont fixées par négociation à l'Assemblée générale (avec des possibilités de renégociations). Les membres permanents du Conseil de sécurité et les autres grands bailleurs de fonds de l'ONU en tirent un poids considérable dans les consultations informelles consacrées à la création d'une opération de paix, en particulier en ce qu'ils peuvent utiliser l'arriéré de paiement comme moyen de pression sur leurs partenaires et sur leurs interlocuteurs du Secrétariat de l'ONU ${ }^{44}$.

A travers ce rapide panorama, nous voulons montrer tout l'intérêt d'une approche empirique soucieuse de retracer les attentes que les délégations membres du Conseil font peser sur celles qui demandent la mobilisation de leurs homologues sur un dossier donné, au regard des positions qui leur sont reconnues dans des groupes précis à des moments précis sur des dossiers précis. De la capacité du « demandeur » à répondre à ces attentes dépendront la reconnaissance et la préservation de positions spécifiques reconnues entre délégations, en vertu de routines interactionnelles institutionnalisées à l'extérieur du Conseil, mais également en vertu de règles propres au jeu diplomatique multilatéral au sein de l'ONU. Parce que souvent informellement reconnues, ces positions peuvent se voir aisément contestées, par petites touches, pour être éventuellement perdues par une délégation, au profit d'une autre le cas échéant.

43 . Jenkins R., op. cit., p. 6.

44. Voir par exemple Novosseloff A., "Les États-Unis et les Nations Unis ", Annuaire français des Relations internationales, 2001, pp. 663-664. 